\title{
Herida penetrante raquimedular por arma blanca: a propósito de dos casos
}

\section{Stab wound injury of the spinal cord: in reference to two cases}

\author{
Raúl de Ramón Silva1, Denisse Finschi Pérez', Gonzalo Diocares Quevedo', Javier Moreno Roco² \\ ${ }^{1}$ Servicio de Neurocirugía, Hospital de Urgencia Asistencia Pública de Santiago de Chile. \\ ${ }^{2}$ Interno VII año, Universidad de Chile.
}

\section{Resumen}

Se describen dos casos operados por el autor: El $1^{\circ}$ un paciente de 30 años que sufrió una agresión por terceros con arma blanca con penetración de columna y médula cervical a nivel de C2-C3, el cual ingresó consciente, tetrapléjico, con un Síndrome de Claude Bernard Horner izquierdo y con compromiso respiratorio leve. El $2^{\circ}$ un paciente de 20 años que también sufrió agresión por terceros con arma blanca con penetración de columna y médula a nivel dorsal, el cual ingresó con compromiso motor distal en L5-S1 izquierda e hipoestesia distal bilateral. En ambos casos se procedió a operar de urgencia para la extracción del elemento extraño, comprobándose que éste transfixiaba la médula en los niveles descritos. En el postoperatorio los pacientes evolucionaron con recuperación parcial de su compromiso neurológico, siendo dados de alta el primero con una hemiplejia izquierda y el segundo solo con un área de hipoestesia distal en la pierna izquierda.

Palabras clave: Trauma raquimedular penetrante por arma blanca, herida por arma blanca penetrante cervical, herida por arma blanca penetrante dorsal.

\section{Abstract}

We described two cases operated by the author: $1^{\circ}$ a 30 -year-old patient who suffered an aggression by third parties with a sharp weapon, with a penetrating injury of the cervical spine and spinal cord at the level of C2-C3, who entered to the hospital tetraplegic, conscious, with a left Claude Bernard Horner syndrome, with mild respiratory compromise. $2^{\circ}$ a 20 -year-old patient who suffered aggression by third parties with a knife, with spinal and spinal cord penetration at the dorsal level, who entered to the hospital with left distal motor compromise in L5-S1 and bilateral distal hypoesthesia. Both were emergency operated to remove the foreign element, verifying during surgery that this was transfixing the spinal cord at the levels described. Postoperative, both patients evolved with partial recovery of their neurological involvement, the first being discharged with a left hemiplegia and the second discharged only with an area of distal hypoesthesia in the left leg.

Key words: Spinal stab wound injury, penetrating spinal cord trauma, cervical penetrating injury, dorsal penetrating injury.

\author{
Correspondencia a: \\ Dr. Raúl De Ramón \\ rdrs62@gmail.com
}




\section{Introducción}

Las lesiones penetrantes raquimedulares por arma blanca son en general lesiones de bastante baja frecuencia, constituyendo en algunas series solo el $10 \%$ de todas las lesiones penetrantes en el raquis, estando el resto generadas en su mayor parte por lesiones por armas de fuego, secundarias a accidentes y en una minoría de tipo autoinferidas ${ }^{1}$. La mayoría de ellas son producto de agresiones por terceros, predominando en el sexo masculino y en el segmento joven de la población.

Dado el compromiso neurológico que conllevan, que dependen del nivel comprometido, suelen ser de alta gravedad, con una morbimortalidad importante y con un alto índice de secuelas. Por otro lado, su escasa frecuencia no permite en general que los neurocirujanos puedan adquirir una adecuada experiencia en su manejo.

Se presentan en esta publicación, dos casos de herida por arma blanca penetrante raquimedular, una a nivel cervical y la otra a nivel dorsal, para exponer su manejo y evolución en el servicio de urgencia.

\section{Casos clínicos}

\section{Primer caso}

Se describe el caso de un paciente de 30 años, de sexo masculino, que fue agredido por terceros en el contexto de privación de libertad, con un objeto contuso cortante. La agresión la recibió en la región cervical posterior, donde esta arma penetró a la altura de C2-C3, transfixiando la columna cervical y la médula, quedando su extremo anterior a nivel cervical anterolateral.

Ingresó al servicio de urgencia del Hospital de Urgencia Asistencia Pública (HUAP), a las pocas horas de sucedido el evento, con el arma in situ (Figura 1), hemodinámicamente estable, con dificultad respiratoria leve, vigil, agitado, con un síndrome de Claude Bernard Horner izquierdo, tetrapléjico y con un nivel sensitivo completo desde C4 hacia distal.

Se le administró profilaxis antitetánica y se procedió a realizar un estudio imagenológico con radiografía simple $(\mathrm{Rx})$ (Figura 2) y con una tomografía axial computarizada (TAC) de columna cervical, los cuales demostraron el arma ingresando en forma parcialmente oblicua de posterior a anterior y de derecha a izquierda a nivel cervical, transfixiando en su totalidad el canal raquídeo y la médula, con el extremo anterior alojado en la región cervical anterior y lateral.

Dados los artefactos producidos por el elemento metálico, no se logró visualizar adecuadamente en el TAC practicado, el compromiso medular secundario ocasionado por el arma.

El paciente fue llevado de forma urgente al pabellón quirúrgico donde debió ser intubado en decúbito lateral, porque el extremo posterior del arma impedía su posicionamiento en decúbito dorsal.

Se operó en decúbito prono, efectuándose una laminectomía de C1 a C3 rodeando el arma, apreciándose en el intra operatorio que traspasaba completamente la duramadre. Al abrir ésta siguiendo el desgarro dural, se observó que el arma penetraba la médula cervical atravesándola también en su totalidad (Figura 3). Se procedió a retirar cuidadosamente el elemento agresor, evidenciándose abundante sangramiento desde la porción anterolateral del canal raquídeo, el cual se

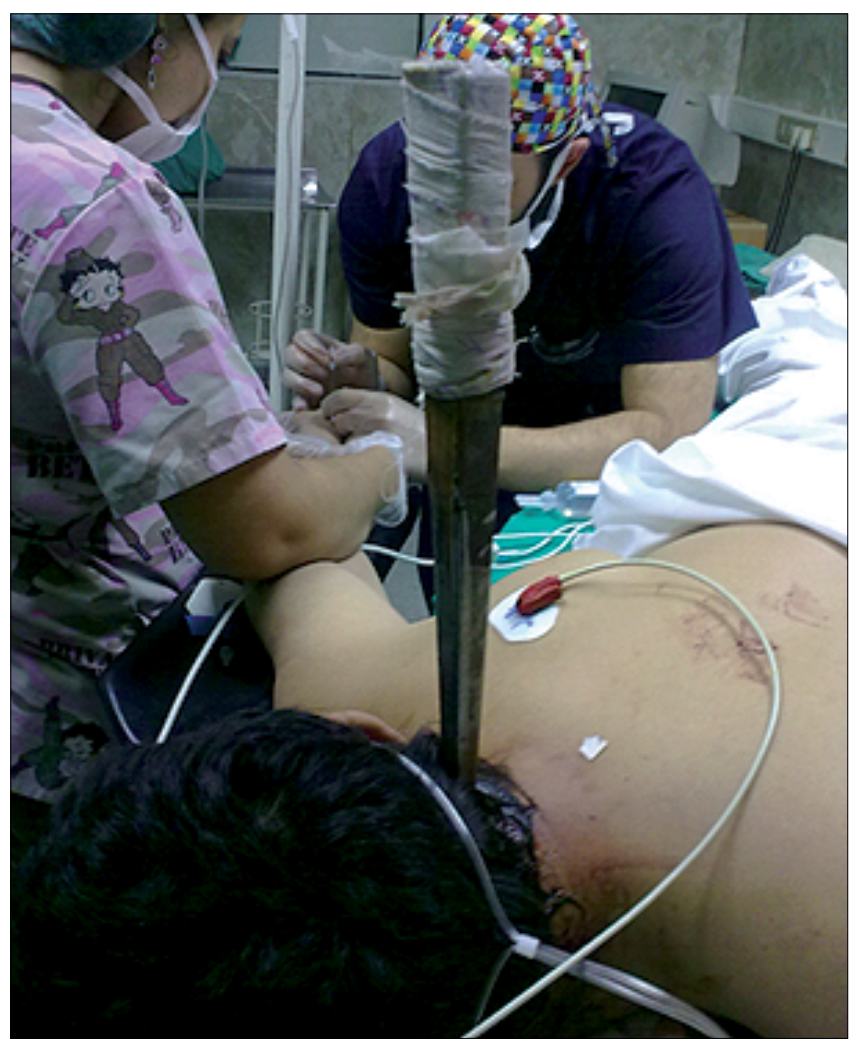

Figura 1. Paciente en decúbito prono con arma blanca in situ a nivel cervical alto con ingreso posterior.

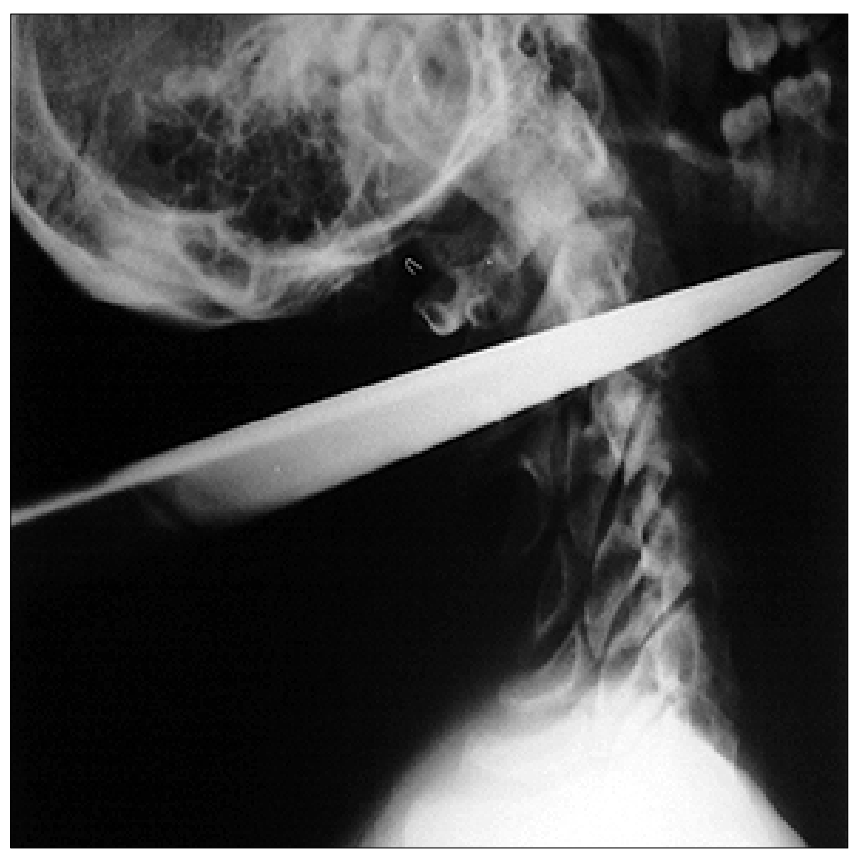

Figura 2. Rx de columna cervical lateral que evidencia compromiso transfixiante de arma blanca a nivel de C2-C3. 


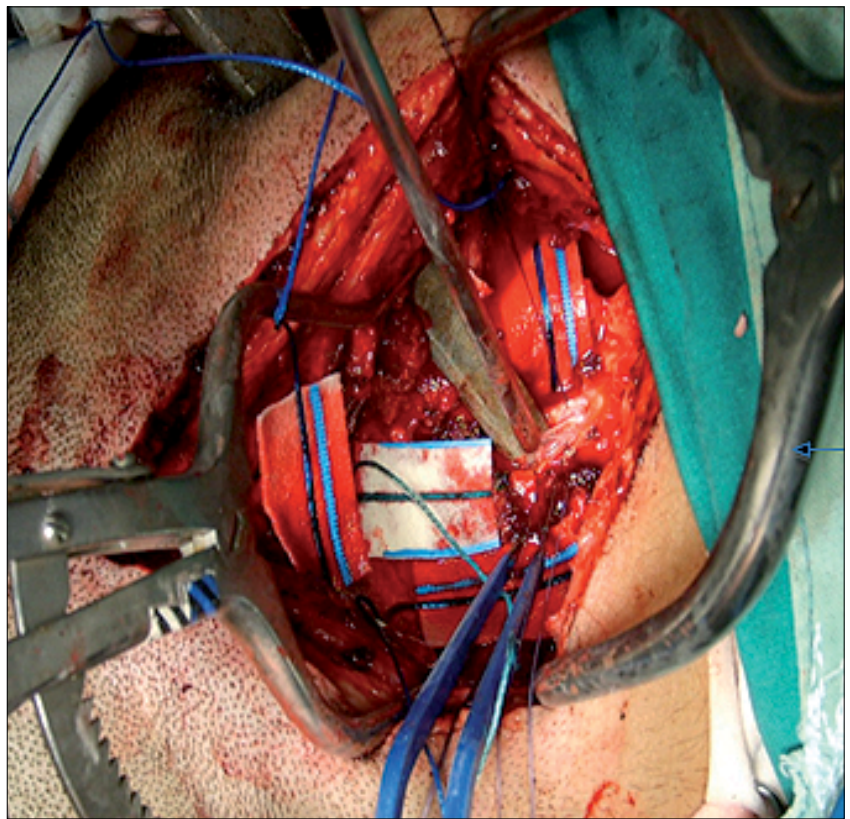

Figura 3. Imagen intraoperatoria con laminectomía de C1 a C3 ya efectuada, donde se aprecia arma blanca in situ transfixiando el cordón medular (flecha).

logró yugular con hemostáticos, coagulación y lavado con abundante suero fisiológico de la zona. Se procedió a efectuar una durorrafia hermética, a completar la hemostasia y a dejar un drenaje extradural.

El enfermo evolucionó con recuperación parcial de su compromiso neurológico, egresando con una hemiplejia izquierda, sin compromiso respiratorio y manteniendo el síndrome de Claude Bernard Horner izquierdo.

En su seguimiento posterior en el centro de origen, el paciente fue dado de alta en buenas condiciones, con la hemiplejia izquierda descrita.

\section{Segundo caso}

Paciente de 20 años, de sexo masculino, que fue agredido también por terceros, en el contexto de privación de libertad, con un cuchillo. La agresión la recibió en la región dorsal donde el arma penetró a la altura de D6-D7, transfixiando tanto la columna como la médula dorsal.

Ingresó al Servicio de Urgencia del HUAP, con el arma in situ (Figura 4) a las pocas horas del evento, hemodinamicamente estable, vigil, sin dificultad respiratoria, con un nivel de disestesia dolorosa en D9, con paresia de L5-S1 izquierda, con hipoestesia de L3 a S1 a derecha y de L5 a S1 a izquierda. A su llegada se le administró profilaxis antitetánica.

Las radiografías y el TAC de columna dorsal demostraron el cuchillo transfixiante a nivel de D6-D7, con compromiso de la médula e impactado en la porción posterior del cuerpo vertebral a ese nivel (Figura $5 a$ y $5 b$ ).

El paciente fue llevado en forma urgente al pabellón quirúrgico, donde se procedió a realizar la laminectomía de D6 y parcial de D7, encontrándose que el cuchillo penetraba la duramadre. Al abrir ésta, el arma atravesaba completamente la médula, encontrándose impactada en la cara posterior del

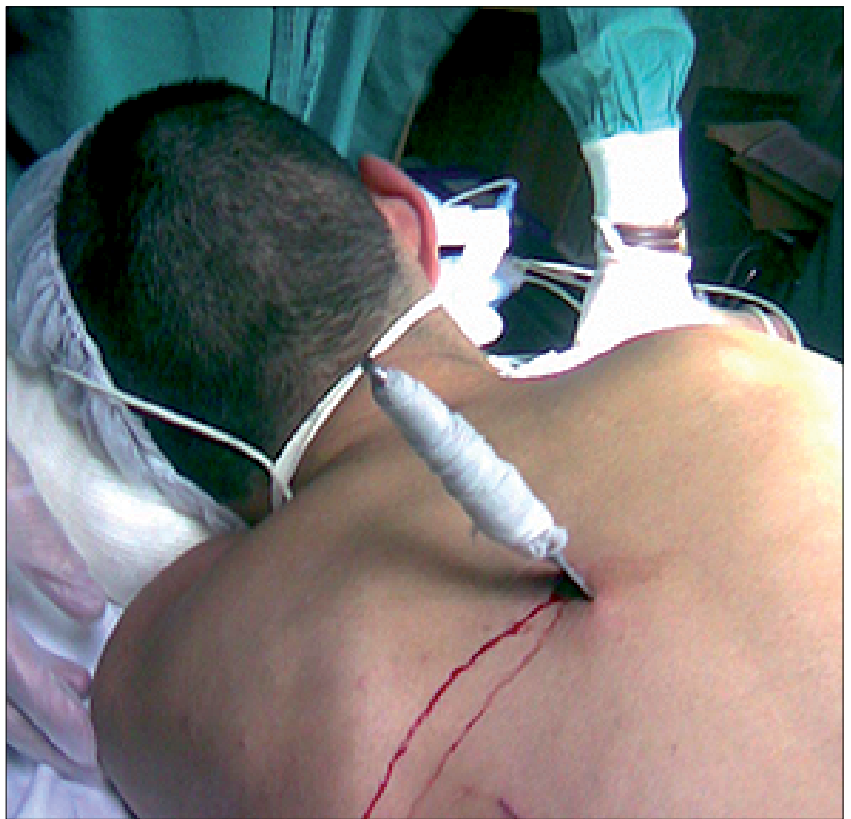

Figura 4. Paciente en decúbito prono con arma blanca in situ a nivel dorsal alto.

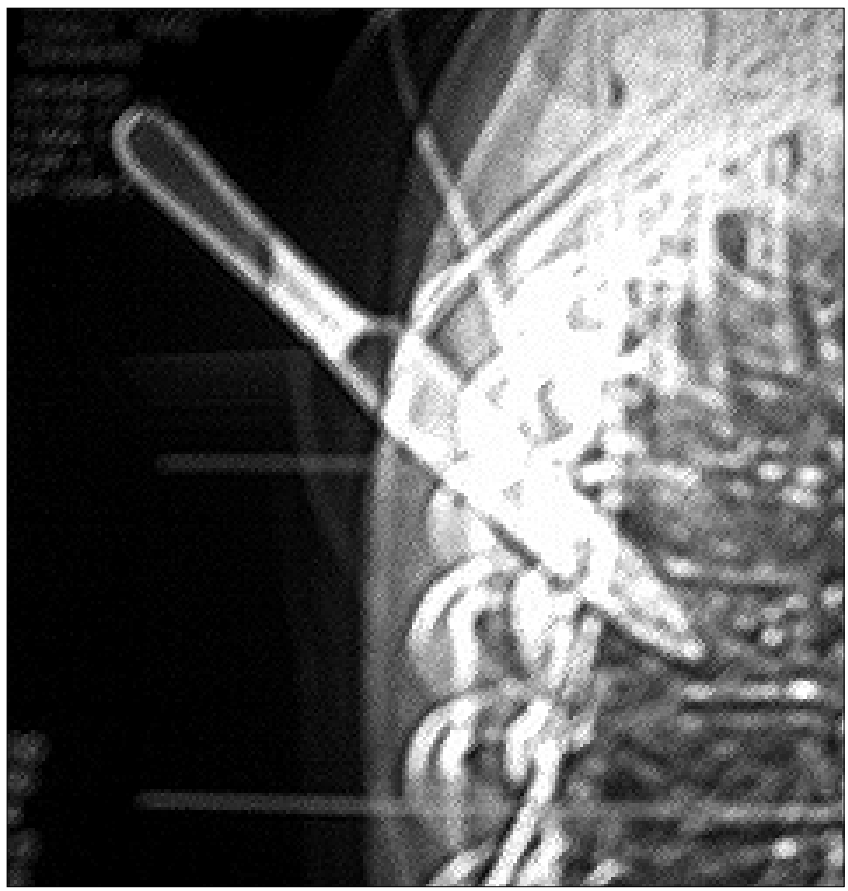

Figura 5a. Rx de columna dorsal lateral con arma in situ.

cuerpo vertebral, lo que ocasionó dificultad para retirarla. Luego de la extracción de ésta no se apreció sangramiento (Figura 6), procediéndose al lavado profuso de la zona y al cierre hermético de la duramadre. Se manejó como en el primer caso con drenaje extradural.

El paciente evolucionó con recuperación completa del compromiso motor y de la disestesia, quedando solo con una zona de hipoestesia a nivel de L5-S1 izquierda al momento 


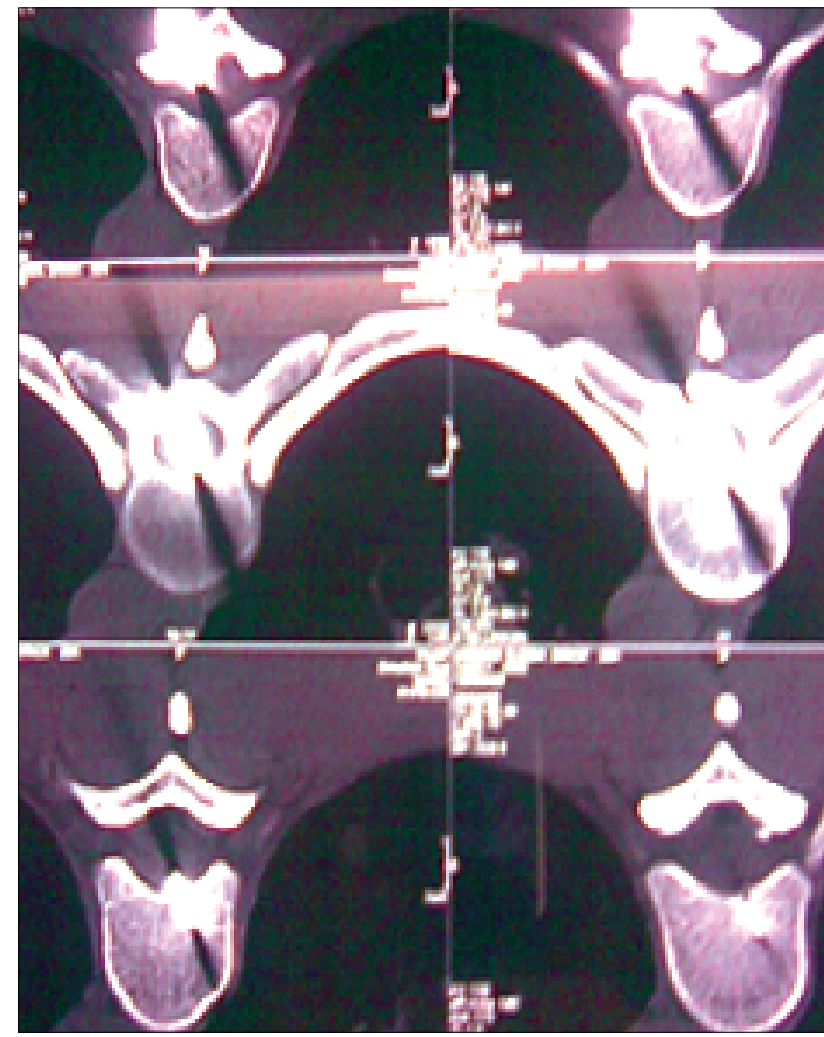

Figura 5b. TAC de columna dorsal axial con ventana ósea que evidencia la profundidad de penetración del arma blanca y su ocupación del canal raquídeo a nivel de D6-D7.

del alta.

En su seguimiento posterior en el centro de origen, el paciente fue dado de alta en buenas condiciones, sin cambios en su condición neurológica y sin complicaciones ulteriores.

\section{Discusión}

Las lesiones penetrantes raqui medulares por arma blanca, constituyen en general una patología de baja incidencia, pero que pueden cursar con graves repercusiones neurológicas, sobre todo cuando se producen a nivel cervical o a nivel dorsal. Son por lo mismo, causantes de una alta morbimortalidad y generadoras de secuelas neurológicas invalidantes cuando se producen a estos niveles.

En relación a su incidencia, tienen una mayor frecuencia en el sexo masculino y en los segmentos jóvenes de la población ${ }^{2,3}$. Del mismo modo son más frecuentes las que se producen a nivel dorsal con $64 \%$ y las que se producen a nivel cervical con $30 \%$, siendo las menos frecuentes las encontradas a nivel lumbar ${ }^{1}$

Las lesiones de la unión cráneo-cervical son extremadamente infrecuentes por la protección que ejerce la escama del occipital y el arco posterior de C1. Cuando estas efectivamente se producen, cursan con altísima mortalidad.

La estructura anatómica de la columna vertebral favorece que los elementos agresores penetren más bien de forma oblicua, dada la presencia de las apófisis espinosas,

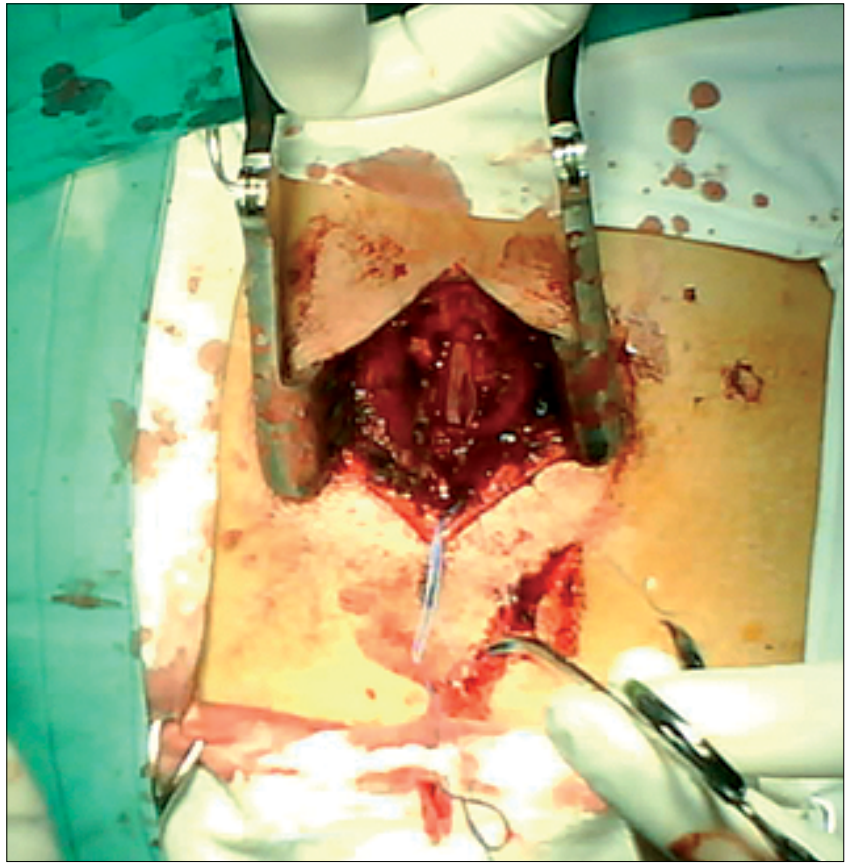

Figura 6. Imagen intraoperatoria luego de la extracción del arma blanca. Se aprecia duramadre y cordón medular con sección lineal transfixiante (flecha).

aportando también protección las apófisis transversas ${ }^{4}$. Esto hace que se den preferentemente lesiones medulares de tipo incompleta. A nivel dorsal la posición anatómica de las láminas con tendencia a estar sobrepuestas puede ocasionar que se produzcan fragmentos óseos que penetren intracanal al ser impactadas por el arma ${ }^{1}$.

Las circunstancias en las que se producen estas lesiones son en primer lugar por agresiones, menos frecuentemente producto de accidentes y en raras circunstancias autoinferidas.

El daño neurológico con que cursan es habitualmente de tipo primario, generado por daño directo y constituido de inmediato desde el inicio del evento. En los casos en que se producen deterioros neurológicos más tardíos, estos suelen obedecer más bien a lesiones de tipo vascular o de tipo infeccioso. También pueden generarse, en caso de desplazamiento de fragmentos óseos, de aparición de edema y por contusión medular ${ }^{5}$.

Este tipo de lesiones medulares suelen ser en su mayor parte de tipo incompleta y en menor proporción de tipo completa. Las lesiones raquimedulares a nivel cervical son las de mayor gravedad. Aquellas sobre C4 pueden cursar con una falla respiratoria, la cual podría no producirse en los casos en que solo hay una hemisección medular por conservación de la función de un hemidiafragma.

Es claro que el tipo de lesión en la médula no solo depende de la altura, sino también de la forma en que el arma atraviesa la estructura medular, lo que incide en los distintos cuadros neurológicos que se objetivan en el examen de estos pacientes. Existen casos en que si el enfermo tiene la fortuna de que su lesión penetre por la línea media en la médula, puedan quedar con pocas secuelas neurológicas, como ocurrió en nuestro segundo caso. 
Si bien este tipo de lesiones son por daño directo del arma, pueden producirse secundariamente lesiones por fragmentos óseos desplazados, por contusión medular y por daño de las estructuras vasculares de la médula con el infarto medular consecutivo ${ }^{1}$.

Clínicamente cuando son a nivel cervical, pueden fácilmente ocasionar la muerte del paciente por falla respiratoria. Del mismo modo, a este nivel pueden cursar con una tetraplejia o una tetraparesia incompleta, con un síndrome de Brown-Séquard según el trayecto de la lesión, con un Claude Bernard Horner por compromiso de la vía simpática, con incidencia clara de requerir ventilación mecánica cuando la lesión cervical es alta (sobre C4) por compromiso diafragmático ${ }^{6}$. Las lesiones a nivel dorsal pueden cursar con una paraplejia o paraparesia incompleta, con un síndrome de hemisección medular y con distintos niveles de compromiso sensitivo tanto superficial como profundo, también relacionados con el trayecto de la lesión. A nivel lumbar por otro lado, pueden producir lesiones radiculares y del cono medular y su curso suele ser bastante más benigno que en las lesiones más altas. En todos estos niveles los enfermos pueden tener asociado compromiso esfinteriano. Pueden también cursar con shock medular inicial y con compromiso hemodinámico secundario $^{7}$, dependiendo del tipo de lesión, como así mismo presentar lesión de otros órganos y estructuras según el nivel en que son infligidas. Otros de los problemas asociados con este tipo de lesiones son la generación de fístulas de líquido cefalorraquídeo (LCR) y las infecciones secundarias como meningitis y aparición de abcesos intra y extra canal, dado el carácter contaminado de los elementos utilizados como armas.

La incidencia de aparición de fístulas de LCR, es relativamente baja en estos enfermos, yendo del orden del $3 \%$ al $4 \%$ de los casos, siendo posible que se produzca su cierre espontáneo, dada la profundidad en la que se ubican las lesiones y que las trayectorias suelen ser de tipo oblicuas y por lo mismo alejadas de la superficie ${ }^{1,4}$.

También pueden producirse como complicación, la presencia de hematomas yuxtadurales, tanto epidurales como subdurales agudos, los que deben ser rápidamente identificados y evacuados si se asocian a compresión medular secundaria ${ }^{8}$.

Es importante también considerar que se pueden producir daños en otras estructuras importantes de la economía como esófago, vía aérea, pulmón, neumotórax, hemotórax, lesión de vasos arteriales y venosos cervicales, lesiones de aorta, lesiones cardiacas, así como de vísceras abdominales y/o retroperitoneales, las cuales deben ser identificadas y tratadas concomitantemente con carácter de urgencia.

En cuanto al estudio imagenológico, hay que separar aquellos enfermos que vienen con el arma in situ de aquellos que no. En los primeros, son de utilidad el estudio radiológico simple asociado a un TAC de columna, considerando que se producirán muchos artefactos dados por la presencia del elemento metálico en este último. En los segundos, idealmente se debe realizar un estudio con Resonancia Nuclear Magnética (RNM) que puede mostrar el tipo de lesión, el trayecto de ésta (hiperseñal en T2), la presencia de colecciones intracanal, de elementos compresivos sobre la médula, la presencia de edema, así como de contusión y de hemorragia intramedular (el poder diferenciar entre edema, contusión, hemorragia y sección medular, permite establecer un pronóstico en estas lesiones). En relación a lo anterior, la ausencia de cambios de la señal intramedular se describe asociado a un mejor pronóstico en algunos trabajos ${ }^{1}$. La presencia de aire intracanal confirma la penetración al canal raquídeo. El estudio se debe complementar además con una angio tomografía (angio-TAC) en los casos de sospecha de lesiones vasculares.

Dentro de la técnica quirúrgica es importante destacar que cuando llegan con el arma in situ, el manejo de ésta debe hacerse obligatoriamente en el intraoperatorio y nunca debe ser retirada si no es en el acto quirúrgico, para controlar potenciales focos de sangrado y tener visión directa para no agravar el daño medular al extraerla.

En caso de que el paciente no llegue con el arma que le ocasionó el daño, el manejo de esos pacientes se describe como controversial en cuanto a la indicación quirúrgica, siendo manejados en muchas ocasiones sólo de manera conservadora. En estos casos, de nuestro punto de vista se sugiere una conducta quirúrgica cuando existen elementos extraños o fragmentos óseos compresivos intracanal (con lesión medular incompleta), en casos de presencia de hematomas yuxtadurales compresivos, en casos de fístula de LCR persistentes y de evidencia de colecciones abscedadas.

Es también aceptado, que el uso de corticoides no estaría indicado en el tratamiento de estos enfermos, ya que no producirían claros beneficios en la evolución y se relacionarían con un aumento en la incidencia de la tasa de infecciones ${ }^{9,10}$.

A diferencia de las lesiones por arma de fuego, las producidas por arma blanca suelen tener una evolución más favorable desde el punto de vista neurológico, dado que no presentan daño por la onda expansiva y de tipo contusivo sobre la médula como se ve en las primeras.

También desde el punto de vista clínico hay que tener en consideración, que los hallazgos neurológicos iniciales suelen ser habitualmente definitivos por la lesión primaria, pero que en la evolución de estos enfermos pueden producirse cambios neurológicos favorables, los que podrían ser explicados por la incidencia de fenómenos contusivos, de edema, por el retiro de elementos compresivos y eventualmente por reversión del shock medular inicial.

En el caso de nuestro primer enfermo, no tenemos una explicación clara de la regresión de su compromiso neurológico de una tetraplejia a una hemiplejia, pudiendo haber incidido en su evolución, cualquiera de los factores anteriormente referidos, especialmente lo relacionado con la oblicuidad de la lesión.

\section{Conclusiones}

Las lesiones penetrantes raquimedulares por arma blanca, son en general lesiones de baja incidencia, pero que se pueden acompañar de una alta morbimortalidad y secuelas, sobre todo cuando se producen en los niveles altos del raquis. El daño neurológico suele establecerse desde un inicio por lesión directa, pero puede tener regresiones si se ha acompañado de fenómenos contusivos, edema y por el retiro del arma causante de la injuria. En general son más 
frecuentes las que cursan con daño medular incompleto. Su incidencia es claramente mayor en el sexo masculino, en la población joven y relacionadas principalmente con agresiones por terceros. Ocurren con mayor frecuencia en la región dorsal y luego en el nivel cervical. Su pronóstico es mucho más favorable que en aquellas lesiones producidas por arma de fuego, ya que no tiene involucrado el daño por la onda expansiva y por la gran contusión que producen estas lesiones. Claramente, hay que diferenciar en su manejo, aquellos pacientes que llegan con el arma in situ de aquellos pacientes que no la traen. En los primeros la conducta es quirúrgica y de urgencia, debiendo retirarse el arma en pabellón para controlar sangramientos y minimizar el daño al extraerla. En los segundos la conducta es más controversial, dependiendo la indicación quirúrgica de la presencia de factores como elementos que causan compresión medular (en las lesiones medulares incompletas), de la presencia de fístula de LCR y si ésta se hace persistente, de la presencia de hematomas yuxtadurales de tipo compresivo y de la aparición de colecciones abcedadas tanto intra como extracanal entre otros. En muchos casos, en ausencia de estos factores, el manejo puede ser conservador y relacionado con la evolución de los pacientes. La presencia de fístula de LCR no necesariamente se asocia a una conducta quirúrgica, ya que por la profundidad de estas lesiones y que suelen ser de tipo oblicuas, pueden cerrarse espontáneamente. En cuanto al estudio imagenológico cuando el arma aún está in situ, son de utilidad el estudio radiológico simple y el TAC de columna, siendo indispensable agregar a lo anterior el estudio con angioTAC cuando se sospecha una probable lesión vascular asociada. En los casos que llegan sin el arma, el estudio de elección debe ser la RNM ya que es la que aporta la mayor información de los daños establecidos y la presencia de complicaciones. No debe olvidarse que este tipo de lesiones pueden asociarse con el daño de otros órganos importantes de la economía, los que deben ser identificados y tratados precozmente ya que pueden cursar por si mismos con morbimortalidad asociada. El uso de corticoides no representaría clara utilidad en estos enfermos y podría aumentar la tasa de incidencia de infecciones. Queda claro por tanto, que este tipo de lesiones son de baja incidencia, por lo que su manejo debe ser enfocado a cada caso en particular, teniendo en consideración todo lo anteriormente descrito.

\section{Referencias}

1. Algorta M, MartínezF. Lesiones medulares por arma blanca. Presentación de casos clínicos y propuesta de manejo. Rev. urug. med. interna. ISSN: 2393-6797 - Mayo 2017 N¹: 39-48.

2. Ropper AE, Neal MT, Theodore N. Acute management of traumatic cervical spinal cord injury. Pract Neurol 2015;15:266272.

3. Shank CD, Walters BC, Hadley MN. Management of acute traumatic spinal cord injuries. Handb Clin Neurol. 2017;140:275-298. doi: 10.1016B978-0-444-63600-3.00015-5.

4. Peacock WJ, Shrosbree RD, Key AG. A Review of 450 Stabwounds of the spinal cord. S Afr Med J. 1977 Jun 25;51(26):961-4.

5. Alizadeh A, Dyck SM and Karimi-Abdolrezaee S. Traumatic Spinal Cord Injury: An Overview of Pathophysiology, Models and Acute Injury Mechanisms. Front. Neurol. 10:282. (2019). doi: 10.3389/fneur.2019.00282

6. Romero-Ganuza FJ, Gambarrutta-Malfatti C, Diez de la LastraBuigues E, Marín-Ruiz MÁ, Merlo-González VE, SánchezAranzueque Pantoja AM, García-Moreno FJ. y Mazaira-Álvarez J. Marcapasos diafragmático como alternativa a la ventilación mecánica en el paciente con lesión medular cervical. Med. Intensiva vol.35 no.1 ene./feb. 2011.

7. Kumar N, Osman A, Chowdhury JR. Traumatic spinal cord injuries. J Clin Orthop Trauma. 2017;8(2):116-124. doi:10.1016/j. jcot.2017.06.022

8. Epstein NE, Hollingsworth R. Diagnosis and management of traumatic cervical central spinal cord injury: A review. Surg Neurol Int 2015;6:S140-53.

9. Levy ML, Gans W, Wijesinghe HS, SooHoo WE, Adkins RH, Stillerman $\mathrm{CB}$. Use of methylprednisolone as an adjunct in the management of patients with penetrating spinal cord injury: Outcome analysis. Neurosurgery 1996; 39:1141-1149.

10. Postigo TR, Rev. Med. Clin. Condes - 2006; 17(1): 12 - 9. Metilprednisolo na en el tratamiento del trauma raquimedular. Análisis de la evidencia. 